\title{
Design of an Innovative Advanced Gastric Simulator
}

\author{
Melita Hribar ${ }^{1}$, Jurij Trontelj ${ }^{1 *}$, Sandra Berglez ${ }^{2}$, Alenka Bevc ${ }^{2}$, Lovro Kuščer ${ }^{3}$, Janez Diaci ${ }^{3}$, and Igor \\ Legen ${ }^{2}$ \\ ${ }^{1}$ University of Ljubljana, Faculty of Pharmacy, Ljubljana, Slovenia \\ ${ }^{2}$ Lek Pharmaceuticals d.d., Ljubljana, Slovenia \\ ${ }^{3}$ University of Ljubljana, Faculty of Mechanical Engineering, Ljubljana, Slovenia
}

\section{ABSTRACT}

This paper presents a newly developed innovative dissolution apparatus with the ability to combine a special pattern of movement, closely mimicking the peristaltic contractions of the human stomach, and the gastric emptying process. The main benefits of the advanced gastric simulator (AGS) are the close physical similarities (shape, volume, and inner wall structure) to the human stomach. The fluid volume, gastric flow, and medium composition can be closely matched to actual in vivo conditions. Moreover, the soft AGS wall is surrounded by a series of constriction mechanisms, which produce the mechanical forces necessary to create the hydrodynamic conditions and physical pressure on its contents aimed at replicating the in vivo conditions. The gastric emptying is enabled by a unique feature, a custom pylorus, which controls the flow of fluids out of the container. Tablets containing a biopharmaceutics classification system (BCS) class III drug were tested to show the applicability of this model. Experiments were performed with the AGS utilizing a United States Pharmacopoeia (USP) apparatus 2. Results demonstrated that mechanical influences on the dosage form in the AGS could be varied by changing the programs of the constriction mechanisms and the settings of the simulated pylorus. The model parameters can be successfully controlled to influence the drug release kinetics.

KEYWORDS: Dissolution, alternative dissolution method, gastric simulator, gastric motility, gastric emptying

\section{INTRODUCTION}

$\mathrm{M}$ any researchers in the drug development field face the challenge of developing the most appropriate in vitro dissolution method and reaching a powerful in vitro-in vivo correlation (IVIVC). The determination of the most suitable conditions needs to be considered. Although the most commonly used classical dissolution tests, USP apparatuses 1 and 2, are widely used especially in quality control, they do not represent realistic in vivo conditions. Constant $\mathrm{pH}$ value, large volumes of dissolution media (900-1000 mL), and a lack of realistic mechanical influences together with unsuitable hydrodynamics can lead to poor interpretation of the results and therefore to low predictive power for the dissolution process in vivo (1).

Considering the fact that over $80 \%$ of pharmaceutical products are given per os, it is important to know the parameters which could affect in vivo drug dissolution (2). After swallowing and passing through the esophagus, a dosage form first enters the stomach, but only a few substances may be absorbed there. Most drugs need to reach their absorption window (i.e., certain segments of the small intestine) where the dissolved drug is then absorbed. Factors such as $\mathrm{pH}$ values of the stomach, the residence time in the stomach, gastric emptying, and fast/ fed conditions are therefore critical for drug absorption in the small intestine (3). In particular, the $\mathrm{pH}$ of the stomach juices, residence time, and dissolution of the drug in the stomach are important for formulations containing weak bases - which are ionized in the stomach and may precipitate after entering the duodenum environment with a higher $\mathrm{pH}-$ and drugs which are highly unstable under acidic conditions. Stomach residence time and dissolution rate in the stomach are important also for formulations with drugs having the absorption window in the upper small intestine together with a short tmax, and controlled-release matrix tablets that may soften in the stomach leading to prompt erosion and dangerous "dose-dumping" (4). Reaching a successful IVIVC can be challenging for immediate-release formulations containing high doses of drugs, especially from BCS classes II and IV, as a sufficient volume of the gastric juices is required to assure the dissolution process (5). Furthermore, building a good model of IVIVC for immediate-release formulations containing very rapidly dissolving BCS class I drugs may be demanding, as the gastric emptying solely dictates the presence of dissolved

* Corresponding author. 
drug at the absorption window in the small intestine. The importance of gastric emptying has been previously highlighted in the literature also for multiple gastroresistant dosage forms, like pellets and minitablets (6). Determination of the important parameters that can significantly affect dissolution after oral application is key for successful prediction of in vivo behavior.

The following details can be considered important when developing a model that should closely resemble the physical appearance of the human stomach. The human stomach is a hollow and elastic organ in the shape of a J. Its normal capacity varies from $0.3 \mathrm{~L}$ in a fasted state to 1.5 L after a meal (7-9). Its shape is influenced by its contents and surrounding organs. An average-sized human stomach is about $30 \mathrm{~cm}$ long, measured along the bottom (greater) curvature, and $10 \mathrm{~cm}$ wide at its widest point after a meal, but collapsed when empty (10). Average fluid volume in a fasted stomach is $45 \mathrm{~mL}$, with a pH between 1 and $3(3,11-13)$. The gastric flow rate as well as secretion rate on average in a fasted state is between 1 and $2 \mathrm{~mL} /$ min (14). Motility under fasting conditions is described as a three-phase migrating motor complex (MMC), lasting 90-120 min when the frequency of individual peristaltic contractions is $2.6 \pm 0.2$ contractions per min, but varies between the phases (15).

Numerous alternative dissolution devices have been invented to explore drug dissolution or food digestion; for example, the glass-bead device, the artificial stomachduodenum model, the dynamic gastric model, the human gastric simulator, and the engineered stomach and small intestine model (16-20). Each model serves to test certain aspects of food degradation or formulation disintegration. The purpose of the present work is to present a novel advanced gastric simulator (AGS) that captures many physical features of an actual human stomach.

To show the usefulness of the novel simulator, an immediate-release formulation containing a high dose of a model drug with high water solubility was chosen. It is classified as a BCS class III drug, as the absorption window is mainly the upper small intestine. The tested model drug has rapid absorption in vivo, indicating that the formulation inside the stomach quickly disintegrates into smaller particles, leading to rapid drug release and gastric emptying under fasted conditions with the ingested fluid. Consequently, the conditions, particularly the stomach residence time and dissolution rate in the stomach, may be extremely important. Therefore, the drug release kinetics of the model drug were observed in our AGS model under different experimental conditions. Modifications of flow rates and contraction intensities, similar to and within the in vivo range, were applied. The results of their influence on the drug release are presented as the first published results obtained with dissolution testing in the newly developed apparatus. There is an increasing need for identifying the impact of gastrointestinal motility and hydrodynamics on formulation properties to develop in vivo predictive dissolution methods (21). The AGS is therefore a powerful new tool, applicable in the area of research and development, to explore the influence of various parameters on drug disintegration and dissolution with any desired degree of similarity to the known in vivo conditions, with the ultimate goal of identifying the crucial factors affecting the drug release.

\section{MATERIALS AND METHODS}

\section{Preparation of the Dosage Form}

Among all solid dosage forms, tablets are the most commonly used for oral application because of their convenience and easy manufacturing (22). Therefore, to show the applicability of the novel apparatus, tablets containing a model drug (BCS class III) were tested by the $\operatorname{AGS}(23)$.

The manufacturers of the excipients are as follows: FMC BioPolymer (USA) for croscarmellose sodium (Ac-Di-Sol) and microcrystalline cellulose (Avicel); Ashland Specialty Ingredients (USA) for hydroxypropyl cellulose (Klucel); FACI SPA (Italy) for magnesium stearate; BASF Care Creations (Germany) for sodium lauryl sulphate (Texapon K12); and Colorcon (USA) for PVA-based coating (Opadry II).

The model drug is an aromatic, heteromonocyclic compound that is soluble in water $(3 \mathrm{mg} / \mathrm{mL})$ and largely soluble in acidic media. Despite the high permeability of the substance, it greatly depends on the absorption enhancing transporters in the proximal gut; therefore, the substance is classified as BCS class III. Following oral administration, the drug has a short tmax value of about $1 \mathrm{~h}$. It is a nucleoside reverse transcriptase inhibitor, so it is used as an antiretroviral drug. Tablets were prepared by the fluid-bed granulation process followed by tablet compression. The average tablet hardness was $125 \mathrm{~N}$ and the drug load was high (> $200 \mathrm{mg}$ ).

\section{Preparation of the Media}

The media, $0.01 \mathrm{M}$ hydrochloric acid $(\mathrm{HCl})$ with the addition of ions $(\mathrm{NaCl})$, was chosen to closely resemble the $\mathrm{pH}$ values of gastric juices in fasted conditions (3). For preparation of $0.01 \mathrm{M} \mathrm{HCl}$ with $\mathrm{NaCl}(2 \mathrm{~g} / \mathrm{L}), 10 \mathrm{~mL}$ of 1 $\mathrm{M} \mathrm{HCl}$ (titrisol $\mathrm{HCl}$, Merck KGaA, Germany) was diluted in $1000 \mathrm{~mL}$ of purified water, after which $2 \mathrm{~g}$ of $\mathrm{NaCl}$ (Merck $\mathrm{KGaA}$ ) was added. 


\section{Dissolution Testing Using USP Apparatus 2}

In vitro dissolution testing was performed using the USP apparatus 2 (708-DS Dissolution Apparatus, Agilent Technologies, Inc., USA) coupled with an automatic sampler (850-DS Dissolution Sampling Station, Agilent Technologies, Inc.) at $50 \mathrm{rpm}$ and $900 \mathrm{~mL}$ of $0.01 \mathrm{M}$ $\mathrm{HCl}$ with $\mathrm{NaCl}(2 \mathrm{~g} / \mathrm{L})$ at $37{ }^{\circ} \mathrm{C} \pm 0.5^{\circ} \mathrm{C}$. The dissolution experiments were performed in three parallels. Samples were taken after $5,10,15,20,25$, and 30 minutes. For each time point, a $1.7-\mathrm{mL}$ sample was automatically collected and filtered through a full flow 1- $\mu \mathrm{m}$ filter (FIL001-EW, Quality Lab Accessories LLC, USA) to 2.0$\mathrm{mL}$ vials. The dissolution medium was not replaced. Samples were diluted with the media 100 -fold prior to injection and analyzed by ultra-high-performance liquid chromatography (UHPLC) system (Waters Corporation, USA). Reagents for the chromatographic assay were purchased from Merck KGaA, Germany; this includes the trimethylamine and orthophosphoric acid. Acetonitrile and methanol were obtained from Avantor Performance Materials, LLC (USA).

\section{Advanced Gastric Simulator (AGS)}

The apparatus, shown in Figure 1, is comprised of a flexible silicone rubber container with eight constriction mechanisms placed around the outer circumference of the container. The container is manufactured by casting up to six layers of silicon rubber (Dragon Skin FX, SmoothOn, Inc., USA) to reach a suitable compromise between preserving the shape in the non-loaded state and the desired shape deformation under a load. The silicone rubber is flexible and soft and exhibits the required chemical inertness. Ridges formed in the longitudinal direction of the interior wall aid in maintaining the shape, and the thin, flexible wall between them facilitates deformation during contraction.

Constriction mechanisms assembled using parts of optical iris diaphragms (57-586, Edmund Optics Inc., USA) allow the inner diameter to be changed, leading to local and annular constriction of the silicone container. Constriction mechanisms are connected to an electric step motor (ST2818, Nanotec Electronic GmbH \& Co. KG, Germany), with plug-in motor controller (SMCP33, Nanotec Electronic $\mathrm{GmbH} \& \mathrm{Co} . \mathrm{KG}$ ), assuring precise and quick adjustment of the aperture. Motor controllers are connected to a computer using a USB/RS485 converter (Brainboxes Ltd., UK). Custom control software has been developed to translate user inputs to programmable motion parameters for motor controllers. The function and structure of constriction mechanisms were described in our previous publication, where a novel intestine model apparatus was introduced (24).

The constriction mechanisms are programmed to change their inner diameter as periodic quasi-sinusoidal functions of time, resulting in corresponding contractions. The contractions are actuated in sequence with a programmable time delay with respect to each other to simulate travelling contraction waves by synchronized opening and closing of the constriction mechanisms.

The apparatus can be used as a closed or open system. In the case of a closed system operation, sampling takes place directly from the silicone container. When used as an open system, samples are taken as aliquots of the flow at the end of the container's outlet. A peristaltic pump (ISMATEC BVP Process IP 65, Cole-Parmer GmbH, Germany) is used to deliver a fresh medium into the container at its inlet at the top opening. At the outlet, a custom valve acts as the pyloric sphincter, which controls the flow of the contents out of the stomach container. The valve is made of two steel pins (PFCLEA-D8-L55-M4, MiSUMi, USA) installed at the container's outlet and connected to the electric step motor (LS2818L0604, Nanotec Electronic GmbH \& Co. KG). The activity of the pyloric valve is controlled by the custom software allowing to set the flow rate (as volume of medium per time unit) through the container's outlet or the pylorus aperture time.

The apparatus is inserted in a temperature-controlled chamber (SP-55 EASY, Kambič d.o.o., Slovenia) to maintain a predetermined temperature (e.g., $37^{\circ} \mathrm{C}$ ).

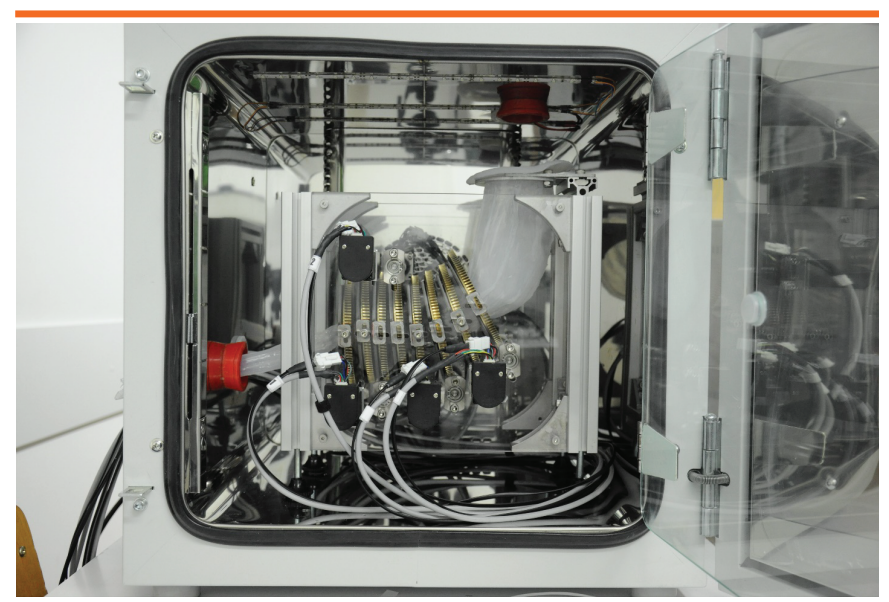

Figure 1. Photograph of apparatus showing the advanced gastric simulator (AGS) inside a temperature-controlled chamber. A silicone stomach container and the constriction mechanisms disposed annularly around the container's outer circumference are shown. For reasons of clarity, the peristaltic pump and the balance are not shown. 


\section{Performing Experiments Using the Advanced Gastric Simulator (AGS)}

A temperature-controlled chamber and medium were preheated to $37^{\circ} \mathrm{C}$. A volume of $250 \mathrm{~mL}$ of medium was poured into the silicone container. Before beginning the experiment, the pyloric valve was closed to prohibit leakage of fluid from the interior of the container. The tablet was applied using long tweezers between the sixth and the seventh constriction mechanism to assure the same starting conditions when performing the experiments.

When performing the closed system experiment, the pyloric valve was completely closed, and the peristaltic pump was not in use. After starting the program of the constriction mechanisms motion, $5 \mathrm{~mL}$ of the sample was collected manually for each time point at the silicone container's top opening. Samples were taken after 5, 10, $15,20,25$, and 30 minutes. The dissolution medium was not replaced. Samples were filtered through a $0.22-\mu \mathrm{m}$ non-sterile, polyvinylidene fluoride (PVDF) syringe filter (Lab Logistics Group GmbH, Germany) into 2.0-mL vials. For the open system experiment, an identical flow rate for the peristaltic pump and the flow through the pyloric valve were chosen. Flow rates of 1 and $2 \mathrm{~mL} / \mathrm{min}$ were tested. At the outlet of the container, the samples were first collected into the small beakers for each sampling point. Samples were taken after 5, 10, 15, 20, 25, and 30 minutes. The samples from each sampling point were further transferred to the syringe and filtered through the $0.22-\mu \mathrm{m}$ filter. All samples were diluted 100 -fold prior to injection and analyzed using UHPLC.

\section{Program Characteristics}

Two different programs, labelled $A$ and $D$, were used. Between programs $A$ and $D$, there is a difference in the period of the mechanism's movement, but the amplitude of constriction mechanisms was the same. Program A provided 3.8 contractions and program $D$ provided 2.3 contractions per minute. The numbers very closely resemble the estimated in vivo contraction rate, which ranges from 2.4-3.6 contractions per minute (25). Cross sectional representation of the travelling contraction wave of program $A$ is presented in Figure 2.

\section{Gastric Emptying Simulation}

The flow of the medium through the simulated pyloric sphincter can be maintained either as a single value for the duration of the experiment or may be altered if needed. Changing the flow rate may offer the possibility of simulating the gastric emptying of fluids, which has been thoroughly studied in humans by different techniques $(12,26)$. To simulate the pattern of gastric emptying according to the in vivo results obtained by a magnetic resonance imaging (MRI) study by Mudie et al. (12), $35 \mathrm{~mL}$ of preheated medium $(0.01 \mathrm{M} \mathrm{HCl}$ with $\mathrm{NaCl}$ $[2 \mathrm{~g} / \mathrm{L}])$ was first added to the stomach container as the resting fluid of a fasted stomach. Next, activity of the constriction mechanisms started (program A) and $240 \mathrm{~mL}$ of water was added. During the experiment, the program for the pyloric valve was adjusted to reach a specific fluid flow rate, as presented in Table 1. A fixed value of the flow rate was set at each time point to reach a steady flow rate during the sequential time points.

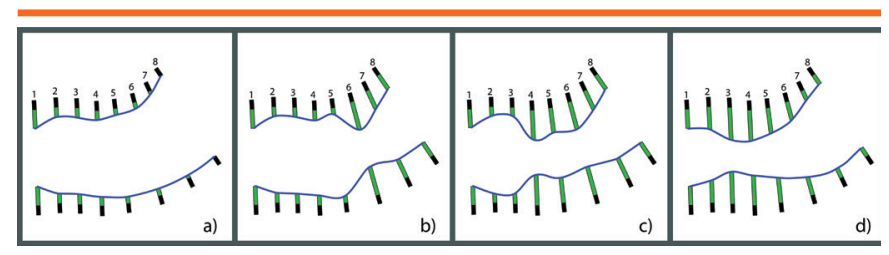

Figure 2. Diagram of travelling contraction wave used in program A shows snapshots of real-time graphic animation from the graphical user interface that illustrate the blade positions (blue line) of the mechanisms at the start of the travelling contraction wave towards the pyloric valve (a) and at three consecutive moments $(b-d)$.

Table 1. Flow Alteration for Simulation of Gastric Emptying.

\begin{tabular}{|c|c|c|}
\hline Time (min) & $\begin{array}{c}\text { Volume of Fluid in } \\
\text { Silicone Container } \\
(\mathrm{mL})\end{array}$ & Flow Rate $(\mathrm{mL} / \mathrm{min})$ \\
\hline- & 35 & - \\
\hline 0 & 275 & 12 \\
\hline 5 & 215 & 15 \\
\hline 10 & 140 & 8 \\
\hline 15 & 100 & 6 \\
\hline 20 & 70 & 4 \\
\hline 25 & 50 & 2 \\
\hline 30 & 40 & 0 \\
\hline
\end{tabular}

\section{Key Features of the AGS}

The shape and size of the full silicone stomach container closely resemble that of a filled adult human stomach; therefore, the behavior and function can be simulated more precisely $(9,10)$. The ridges formed on the interior wall of the container mimic the gastric rugae of the human stomach (10). The silicone container narrows at the pyloric region to $1 \mathrm{~cm}$, which is in accordance with Ferrua et al. who reported that the pyloric ring has a diameter of $1.1 \mathrm{~cm}$ or less (9).

The volume of the media $(250 \mathrm{~mL})$ was chosen to approach more realistic, in vivo conditions compared to the USP apparatus 2 where media volume is normally $500-900$ $\mathrm{mL}$. The volume is similar to the fluid volume after drinking 
one glass of water with the dosage form (27). The $\mathrm{pH}$ of the media was adjusted in relation to the realistic in vivo fasted conditions $(0.01 \mathrm{M} \mathrm{HCl}$ with addition of ions [NaCl]) (3). Optional values of the flow rates in and out of the container can be selected by using the valve and the peristaltic pump, although in vivo relevant flow rates (from 1-5 mL/min) were chosen most frequently (14). The flow rate through the pyloric valve can be adjusted separately from the constriction mechanisms by changing the settings during the experiment. Particular patterns of gastric emptying can be achieved in accordance with Mudie et al. (12).

Constriction mechanisms are mostly present in the lower section of the stomach container where mixing and grinding of the ingredients take place (4). The constriction mechanisms exert pressure on the container's wall, leading to a concentric constriction of the container. The constriction mechanisms influence the dosage form indirectly by providing the physical contact of the silicone container wall and the dosage form. Electric current driving the motor created contraction forces which were measured using the wireless motility capsule and proved to be in agreement with the in vivo data (28, 29). Using an endoscopic camera (VOLTCRAFT BS-10 USB, Conrad Electronic International GmbH \& Co. KG, Germany), actual mechanical disintegration of dosage forms was observed as a consequence of forces exerted by the contraction mechanisms. The container's interior during the contraction is shown in Figure 3. Customized software enables programming patterns of contractions, which can simulate the peristaltic movement of the human stomach. The initial blade position, amplitude, period of blade motion, and the time delay between adjacent constriction mechanisms can be individually set.

\section{RESULTS AND DISSCUSION}

Different experimental conditions were applied using tablets of the model drug to present the applicability of the new AGS apparatus for the model drug. Experimental conditions for simulating the contractions and gastric flow rates were chosen based on published data $(12,14$, 25).

This paper is primarily focused on the description of the newly developed dissolution testing apparatus and a demonstration of its usefulness. The aim of the current work was to present and evaluate parameters that can be varied and therefore might impact the drug release kinetics in this model. It is important for the demonstration of applicability of the new model to highlight the possibilities for varying the experimental conditions, which allow for a range of various movement patterns and also enable an optimization of the method for a specific formulation under investigation.

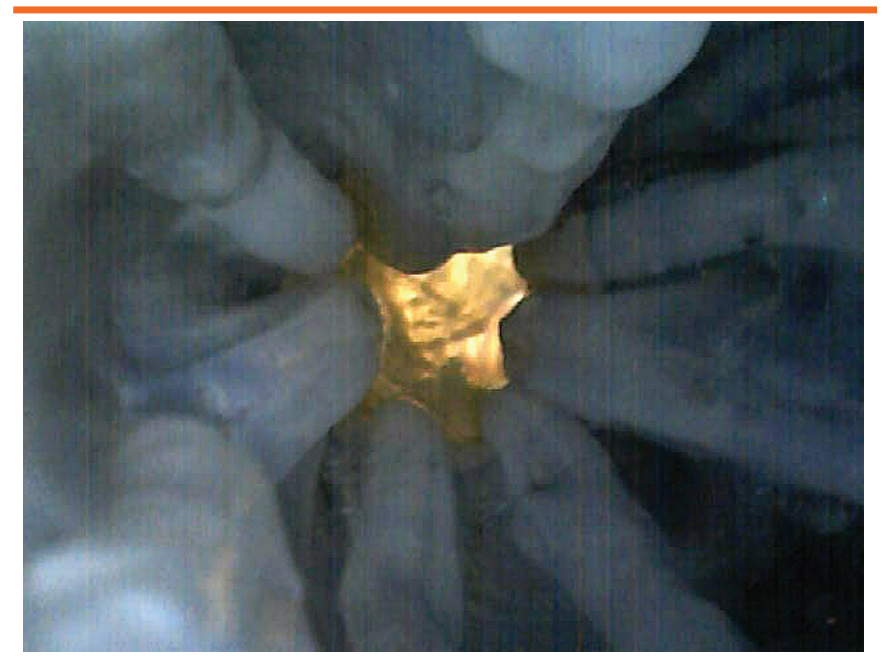

Figure 3. Photograph of the container's interior at the intermediate point of the contraction using the endoscopic camera. Blades forming the constriction mechanism exert pressure on the container's wall, leading to a concentric constriction of the container. Ridges, formed in a longitudinal direction on the interior wall, aid in maintaining the shape, while the thin, flexible wall between them facilitates deformation during contraction. For reasons of clarity, the photograph was taken without the medium inside the container.

\section{Comparison of Closed vs. Open System Experiments on Drug Release from Tablets}

As immediate-release formulations, which contain very rapidly dissolving drugs with a short tmax, may prove challenging to achieve a good IVIVC, immediate-release tablets of a model drug were chosen for the experiments. The model drug has an absorption window mostly in the proximal small intestine which means that the stomach conditions in which the dosage form is exposed are highly important. Although immediate-release tablets are believed to be normally mechanically unsusceptible (30), the conditions and kinetics for tablet disintegration are very important from the dissolution point of view. The sample formulation was previously determined as rapidly dissolving in the USP apparatus 2 experiments. In general, fast dissolution profiles of different formulations obtained in classical dissolution tests often lead to lower discriminating power between the samples. A rapidly dissolving drug formulated in an immediate-release formulation was chosen to compare results from closed and open system experiments for evaluation and possible optimization of the open system. The open system is where the dissolved drug is detected at the outlet of the stomach container, which might be an important sampling point to estimate drug level availability for absorption in the small intestine. 
Figure 4 shows the difference in the drug dissolution profiles by performing two different types of experiments (closed vs open system). When performing the closed system experiment, samples were collected manually at the silicone container's top opening. The drug is very soluble, therefore the dissolution profile obtained in AGS using the closed system indicated a fast dissolution despite using less medium than the USP apparatus 2 experiments. The profile in the AGS indicated a slightly faster dissolution rate compared to the USP apparatus 2 , which is most likely the result of higher mechanical stress caused by the peristaltic movement of the AGS. For the open system experiments, the flow rate through the AGS container was maintained by the regulation of the pyloric valve and the peristaltic pump. Surprisingly, when comparing the dissolution profiles between the closed and open system, the latter showed slower drug release (Fig. 4). Using the open system, the samples were collected cumulatively at the outlet of the container, meaning the dissolved drug at the sampling point was further away from the tablet's initial dissolving location, which leads to the apparent slower drug release rate in the open system experiment. The different sampling point, in addition to the different hydrodynamic conditions and flows inside the container, may explain the slower dissolution rate observed in the open system experiment. The orifice of the simulated pylorus contributed to fluid flowing through the container and shorter contact of media with the dosage form due to fresh media being delivered by a peristaltic pump, whereas in the closed system experiment, the fluid movement is significantly different. During the experiment it was observed that the fluid was greatly pushed back towards the proximal part of the stomach container, which is where the sampling takes place. Open system experiments with a slower apparent drug dissolution rate may be valuable if dissolution tests with greater discriminating power are desired, which would be especially beneficial for evaluating immediate-release dosage forms when the dissolution rate is often so fast that differences between formulations are very hard to detect using conventional dissolution systems. Moreover, open system experiments allow closer simulation of in vivo conditions, similar to the USP apparatus 2 . With continuous flow through the simulated pylorus, the dissolved drug is detected at the outlet of the stomach, which is very likely the most important sampling point for estimation of the drug levels available for absorption further down the gastrointestinal (GI) tract (3).

\section{Influence of Different Programs and Flow Rates on Drug Release from Tablets}

The influence of changing the flow rate results in different dissolution profiles, as presented in Figure 4. The in vivo gastric flow rate in a fasted state depends on the phase of the $\mathrm{MMC}$ and normally reaches the values of up to $2 \mathrm{~mL} / \mathrm{min}$, whereas in the fed state, it increases up to 4

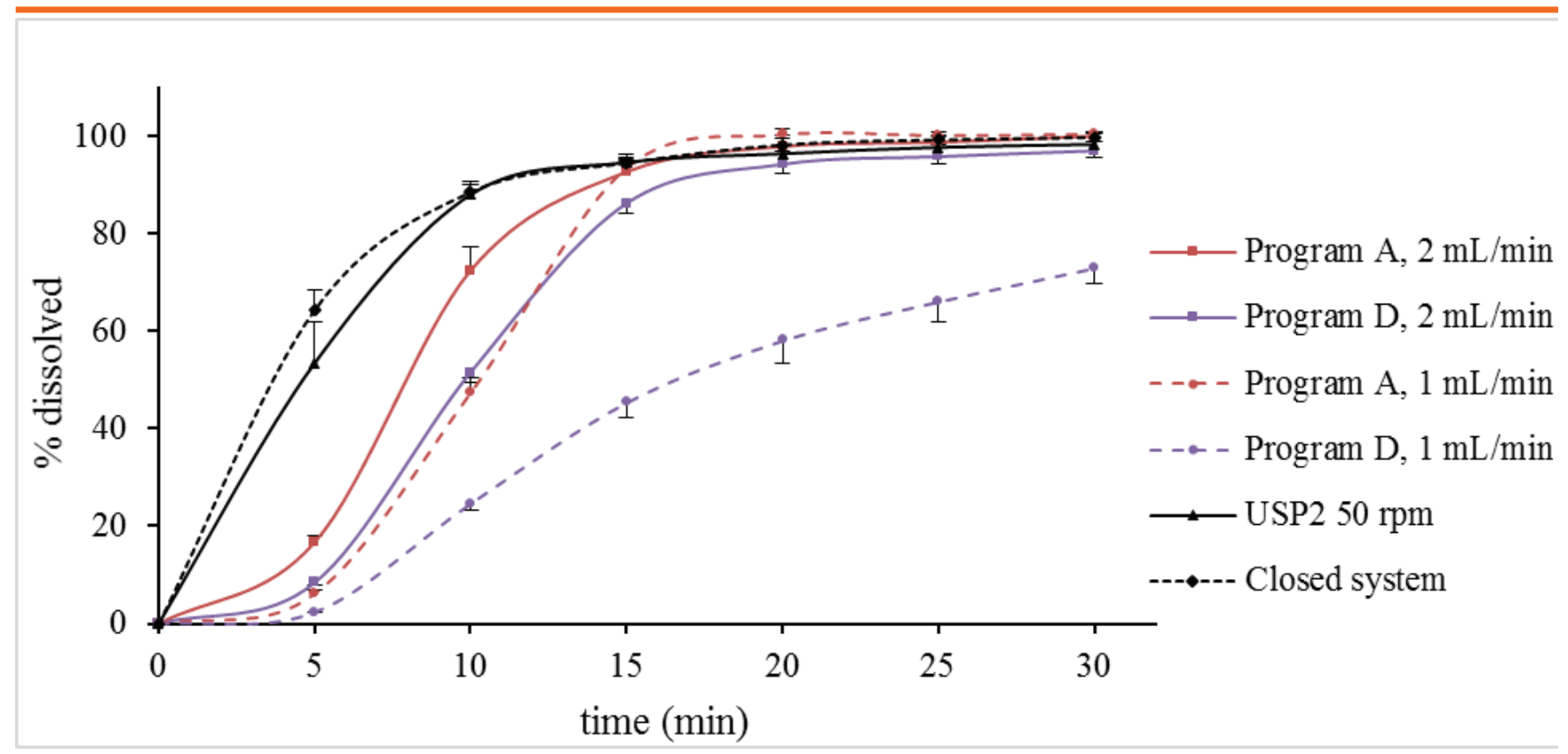

Figure 4. Dissolution profiles for tablets obtained in the advanced gastric simulator (AGS) (n=5) in $0.01 \mathrm{M} \mathrm{HCl} \mathrm{with} \mathrm{NaCl}(2 \mathrm{~g} / \mathrm{L}) \mathrm{compared}$ to the profile obtained in USP apparatus 2 (USP2) at $50 \mathrm{rpm}(n=3)$. Data are shown as mean \pm standard error. 
$\mathrm{mL} / \mathrm{min}(4,14)$. Therefore, testing different flow rates in the AGS experiments may be relevant to explore the possible influences of varying flow rates on the in vitro drug dissolution. The faster program A, providing 3.8 contractions per minute, and the slower program $D$, providing 2.3 contractions per minute, were both tested with the flow rates or 1 and $2 \mathrm{~mL} / \mathrm{min}$. Results obtained in the AGS were slower compared to the USP apparatus 2. As expected, the faster program A led to a faster drug release rate compared to the slower program $D$; however, a greater impact of the two different contraction rates was seen at the slower flow rate $(1 \mathrm{~mL} / \mathrm{min})$ compared to the faster rate $(2 \mathrm{~mL} / \mathrm{min})$. The drug dissolved faster and diffused more rapidly toward the sampling point at the container's outlet due to the increased speed of the peristaltic wave, whereas a slower peristaltic wave and flow rate resulted in a slower release rate. The results showed the possibility to moderate the drug dissolution process. The slower program and flow rate can greatly decrease the drug release rate despite the dissolution profile obtained in the USP apparatus 2 being very fast. It can be speculated that decreasing the speed of the programs can be used in detecting the small differences in dissolution of similar formulations, which may be relevant for absorption kinetics in vivo.

\section{Gastric Emptying Simulation}

The main benefits of AGS, besides the very close physical similarity to the human stomach, are the combination of a special motion similar to the peristaltic movement and a custom pylorus, which enable the creation of a specific fluid flow out of the silicone container. Experiments were performed to reach a comparable curve describing the pattern of gastric emptying of fluid in vivo (12). The result of a programmed alteration of the fluid flow rates (Table 1) and consequently, a decrease in the fluid volume in the silicone container is shown in Figure 5. Immediately after the ingestion of water, the gastric liquid volume in vivo is approximately $90 \%$ of the expected volume due to the wide range of measurements and inter-individual variability. The measured value of the gastric volume is $242 \pm 9 \mathrm{~mL}$ (Fig. 5b). In the in vitro method, the total volume (resting liquid volume and administered volume of water) is precisely $275 \mathrm{~mL}$ (Fig. 5a).

This method is a demonstration of the possibility for in vitro simulation of fasted gastric emptying following ingestion of a glass of water $(240 \mathrm{~mL})$, as recommended for bioavailability or bioequivalence studies. The experiments were performed using only the dissolution medium, however, the concept showed the feasibility to repeatedly achieve dynamic moderation of the fluid flow rate as desired. The opportunity to change the fluid flow

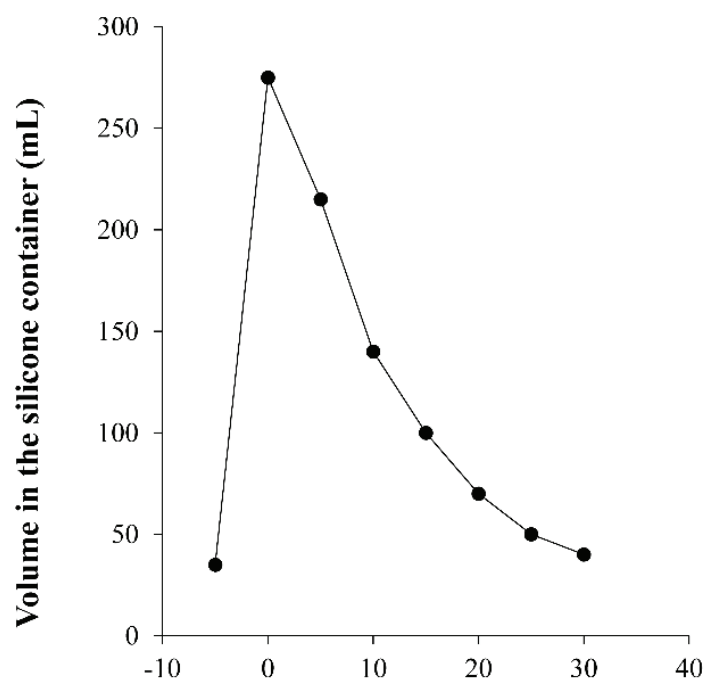

a)

Time (min)

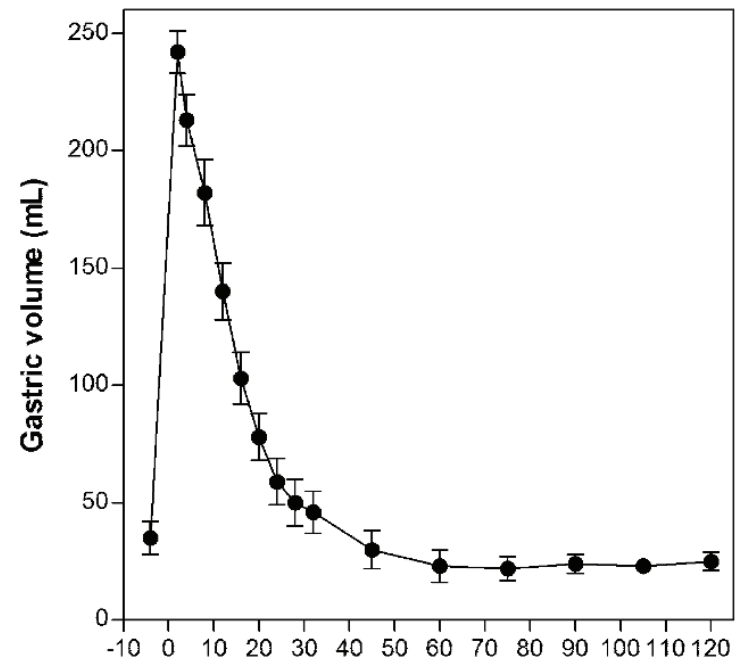

b)
Time (min)

Figure 5. Graph (a) shows the pattern of gastric emptying achieved by adjusting the flow through the simulated pyloric sphincter. Graph (b) shows mean ( \pm standard error) gastric volume obtained from 12 healthy volunteers by MRI; used with permission from (12). Mudie, D. M.; Murray, K.; Hoad, C. L.; Pritchard, S. E.; Garnett, M. C.; Amidon, G. L.; Gowland, P. A.; Spiller, R. C.; Amidon, G. E.; Marciani, L. Quantification of gastrointestinal liquid volumes and distribution following a $240 \mathrm{~mL}$ dose of water in the fasted state. Mol. Pharm. 2014, 11, 3039-3047.

DOI: 10.1021/mp500210c. Copyright 2014. American Chemical Society (ACS). Available under the terms of the ACS AuthorChoice via CC-BY 4.0 License.6. 
rate during the experiment may lead to the development of different experimental methods for simulating the gastric emptying of various food and drinks. Simulation of gastric emptying may offer advantages for exploring the in vitro effects of gastric passage for gastro-resistant and controlled-release dosage forms.

\section{Advantages of the AGS and Future Perspectives}

Although all alternative in vitro models offer the possibility to adjust the volume, temperature, and $\mathrm{pH}$ of various media, none combine anatomical similarities of the human stomach with the possibility to simulate the peristaltic wave pattern and gastric emptying. The conditions of the experiments are specifically set by customized software to be as close as possible to the in vivo conditions based on published data regarding the gastric flow rate and contractions $(12,14,25)$. Moreover, by successfully controlling all the parameters, the model allows us to study crucial parameters separately (e.g., physical influences or gastric emptying of the dosage forms) or adjust the program settings for specific formulations based on the results from clinical studies to achieve a good IVIVC. The closer the similarity between in vitro and in vivo dissolution conditions, the greater the predictive power of a model is considered to be. However, the advantage of reaching a good IVIVC is a goal that still needs to be demonstrated in the future. The data in this manuscript are presented with the objective to describe the performance of the AGS and the possibility of the model parameters' variation, which may influence the drug release kinetics.

Different patterns of peristaltic movement and various intensities of physical contact can be created by changing the programs, therefore the apparatus activity may represent an interesting research tool for food digestion studies. In vitro simulation of the fed stomach can be developed by adding food or, as an example, glass beads to the stomach container causing higher shear stress on the surface of the pharmaceutical dosage forms, which could be especially important for dosage forms like matrix tablets or capsules.

Different gastric transit times, created by changing the flow rate, offer the possibility to explore the gastric emptying of multiple dosage forms (e.g., pellets have a special pattern of entry into the small intestine). Also, the variability of in vivo gastric motility may be simulated, with potentially far reaching benefits for researching the effect of gastric motility of special populations or different pathological conditions on drug dissolution. Moreover, studying gastric conditions is important for evaluating the behavior of drugs that are rapidly dissolved and absorbed in vivo. Last, but not least, the intestine model for simulating the peristaltic action (IMSPA), presented in our previous publication, could serve as a continuation of the present experiment (24). Combination of both apparatuses, the AGS and the IMSPA, may lead to development of a simulation method of the GI tract for passage of controlled-release dosage forms.

\section{CONCLUSIONS}

This paper presents a newly developed gastric simulator for dissolution testing. The novelty of the model is that it is designed to resemble as closely as possible many physiological features of the human stomach. The focus of the current work was to describe the mechanism of action and parameters that can be varied and therefore might impact the drug release kinetics in this model. The applicability of the apparatus was demonstrated for immediate-release tablets containing a model drug classified as BCS class III. Results obtained in the AGS demonstrated that experimental conditions can be repeatedly and successfully controlled to create different movement patterns and flow rates. Moreover, by adjusting the parameters, the model allows us to study crucial parameters separately or moderate program settings for a specific formulation in the drug development process. However, the physiological relevance of the model needs to be further evaluated and confirmed by comparison with in vivo data. Based on the close physical similarity of the model to the in vivo situation, this AGS could become an experimental tool for closer prediction of the in vivo dissolution process compared to conventional dissolution methods, thereby reducing the costs of new drug development and in vivo studies.

\section{ACKNOWLEDGMENTS}

The authors would like to acknowledge Ms. Petra Omerzu and Mr. Ognen Jakasanovski from the Faculty of Pharmacy Ljubljana for assistance in conducting experiments.

This research did not receive any specific grant from funding agencies in the public, commercial, or not-forprofit sectors.

\section{CONFLICTS OF INTEREST}

The authors declared no conflicts of interest related to this article.

\section{REFERENCES}

1. Koziolek, M.; Grimm, M.; Becker, D.; Iordanov, V.; Zou, H.; Shimizu, J.; Wanke, C.; Garbacz, G.; Weitschies, W. Investigation of $\mathrm{pH}$ and temperature profiles in the $\mathrm{Gl}$ tract of fasted human 
subjects using the Intellicap $\left({ }^{\circledR}\right)$ System. J. Pharm. Sci. 2014, 104, 2855-2863. DOI: 10.1002/jps.24274.

2. McConnell, E. L.; Fadda, H. M.; Basit, A. W. Gut instincts: explorations in intestinal physiology and drug delivery. Int. J. Pharm. 2008, 364, 213-226. DOI: 10.1016/j. ijpharm.2008.05.012.

3. Mudie, D. M.; Amidon, G. L.; Amidon, G. E. Physiological parameters for oral delivery and in vitro testing. Mol. Pharm. 2010, 7, 1388-1405. DOI: 10.1021/mp100149j.

4. Koziolek, M.; Garbacz, G.; Neumann, M.; Weitschies, W. Simulating the postprandial stomach: Physiological considerations for dissolution and release testing. Mol. Pharm. 2013, 10, 1610-1622. DOI: 10.1021/mp300604u.

5. Sakore, S.; Chakraborty, B. In vitro-in vivo correlation (IVIVC): A strategic tool in drug development. J. Bioequiv. Availab. 2011, S3. DOI: 10.4172/jbb.S3-001.

6. Locatelli, I.; Nagelj Kovacic, N.; Mrhar, A.; Bogataj, M. Gastric emptying of non-disintegrating solid drug delivery systems in fasted state: relevance to drug dissolution. Expert Opin. Drug Deliv. 2010, 7, 967-976. DOI: 10.1517/17425247.2010.495982.

7. Camilleri, M.; Breen, M.; Ryks, M.; Burton, D. Proximal and overall gastric emptying of solids in patients with reduced gastric volume accommodation compared to matched controls. Dig. Dis. Sci. 2011, 56, 1729-1734 DOI: 10.1007/s10620-011-1615-0.

8. de Zwart, I. M.; de Roos, A. MRI for the evaluation of gastric physiology. Eur. Radiol. 2010, 20, 2609-2616. DOI: 10.1007/ s00330-010-1850-3.

9. Ferrua, M. J.; Singh, R. P. Modeling the fluid dynamics in a human stomach to gain insight of food digestion. J. Food Sci. 2010, 75, R151-R162 DOI: 10.1111/j.1750-3841.2010.01748.x.

10. Schulze, K. Imaging and modelling of digestion in the stomach and the duodenum. Neurogastroenterol. Motil. 2006, 18, 172183. DOI: 10.1111/j.1365-2982.2006.00759.x.

11. Lobo, D. N.; Hendry, P. O.; Rodrigues, G.; Marciani, L.; Totman, J. J.; Wright, J. W.; Preston, T.; Gowland, P.; Spiller, R. C.; Fearon, K. C. H. Gastric emptying of three liquid oral preoperative metabolic preconditioning regimens measured by magnetic resonance imaging in healthy adult volunteers: A randomised double-blind, crossover study. Clin. Nutr. 2009, 28, 636-641. DOI: 10.1016/j. clnu.2009.05.002.

12. Mudie, D. M.; Murray, K.; Hoad, C. L.; Pritchard, S. E.; Garnett, M. C.; Amidon, G. L.; Gowland, P. A.; Spiller, R. C.; Amidon, G. E.; Marciani, L. Quantification of gastrointestinal liquid volumes and distribution following a $240 \mathrm{~mL}$ dose of water in the fasted state. Mol. Pharm. 2014, 11, 3039-3047. DOI: 10.1021/mp500210c.

13. Steingoetter, A.; Fox, M.; Treier, R.; Weishaupt, D.; Marincek, B.; Boesiger, P.; Fried, M.; Schwizer, W. Effects of posture on the physiology of gastric emptying: a magnetic resonance imaging study. Scand. J. Gastroenterol. 2006, 41, 1155-1164. DOI: 10.1080/00365520600610451.

14. Schindlbeck, N. E.; Heinrich, C.; Müller-Lissner, S. A. Relation between fasting antroduodenal motility and transpyloric fluid movements. Am. J. Physiol. 1989, 257, G198-G201. DOI: 10.1152/ajpgi.1989.257.2.G198.

15. de Zwart, I. M.; Mearadji, B.; Lamb, H. J.; Eilers, P. H. C.; Masclee, A. A. M.; de Roos, A.; Kunz, P. Gastric motility: comparison of assessment with real-time MR imaging or barostat measurement initial experience. Radiology 2002, 224, 592-597. DOI: 10.1148/ radiol.2242011412.

16. Bogataj, M.; Cof, G.; Mrhar, A. Development of a glass-bead device for dissolution testing. Dissolution Technol. 2015, 22, 18-24. DOI: 10.14227/DT220315P18.

17. Carino, S. R.; Sperry, D. C.; Hawley, M. Relative bioavailability estimation of carbamazepine crystal forms using an artificial stomach-duodenum model. J. Pharm. Sci. 2006, 95, 116-125. DOI: 10.1002/jps.20495.

18. Wickham, M. J. S.; Faulks, R. M.; Mann, J.; Mandalari, G. The design, operation, and application of a dynamic gastric model. Dissolution Technol. 2012, 19, 15-22. DOI: 10.14227/ DT190312P15.

19. Kong, F.; Singh, R. P. A human gastric simulator (HGS) to study food digestion in human stomach. J. Food Sci. 2010, 75, E627E635. DOI: 10.1111/j.1750-3841.2010.01856.x.

20. Guerra, A.; Denis, S.; le Goff, O.; Sicardi, V.; François, O.; Yao, A.F.; Garrait, G.; Manzi, A. P.; Beyssac, E.; Alric, M.; Blanquet-Diot, S.. Development and validation of a new dynamic computercontrolled model of the human stomach and small intestine. Biotechnol. Bioeng. 2016, 113, 1325-1335. DOI: 10.1002/ bit. 25890 .

21. Lennernäs, H.; Lindahl, A.; Van Peer, A.; Ollier, C.; Flanagan, T.; Lionberger, R.; Nordmark, A.; Yamashita, S.; Yu, L.; Amidon, G. L.; Fischer, V.; Sjögren, E.; Zane, P.; McAllister, M.; Abrahamsson, B. In vivo predictive dissolution (IPD) and biopharmaceutical modeling and simulation: future use of modern approaches and methodologies in a regulatory context. Mol. Pharm. 2017, 14, 1307-1314. DOI: 10.1021/acs.molpharmaceut.6b00824.

22. Pande, V.; Karale, P.; Goje, P.; Mahanavar, S. An overview on emerging trends in immediate release tablet technologies. Austin Ther. 2016, 3, 1026.

23. Legen, I.; Bevc, A.; Berglez, S.; Diaci, J.; Kuscer, L. Apparatus for simulating the function of human stomach and/or human intestine. U.S. Patent 2016/0351079 A1, December 1, 2016. .

24. Hribar, M.; Trontelj, J.; Klančar, U.; Markun, B.; Čeligoj Dujc, T.; Legen, I. A novel intestine model apparatus for drug dissolution capable of simulating the peristaltic action. AAPS PharmSciTech 2017, 18, 1646-1656. DOI: 10.1208/s12249-016-0629-5.

25. Romagnoli, J. M. Collard, R.; Romagnoli, R. Human stomach has a recordable mechanical activity at a rate of about three cycles/minute. Eur. J. Surg. 2001, 167, 188-194. DOI: 10.1080/110241501750099357.

26. Yamashita, S.; Kataoka, M.; Higashino, H.; Sakuma, S.; Sakamoto, T.; Uchimaru, H.; Tsukikawa, H.; Shiramoto, M.; Uchiyama, H.; Tachiki, H.; Irie, S. Measurement of drug concentration in the 
stomach after intragastric administration of drug solution to healthy volunteers: Analysis of intragastric fluid dynamics and drug absorption. Pharm. Res. 2013, 30, 951-958. DOI: 10.1007/ s11095-012-0931-1.

27. Bioavailability and Bioequivalence Studies for OrallyAdministered Drug Products-General Considerations; Guidance for Industry; U.S. Department of Health and Human Services, Food and Drug Administration, Center for Drug Evaluation and Research (CDER), U.S. Government Printing Office: Washington, DC, 2003.

28. Kamba, M.; Seta, Y.; Kusai, A.; Ikeda, M.; Nishimura, K. A unique dosage form to evaluate the mechanical destructive force in the gastrointestinal tract. Int. J. Pharm. 2000, 208, 61-70. DOI: 10.1016/S0378-5173(00)00552-4.

29. Hribar, M.; Jakasanovski, O.; Trontelj, J.; Grabnar, I.; Legen, I. Determining the pressure-generating capacity of the classical and alternative in vitro dissolution methods using a wireless motility capsule. J. Pharm. Innov. 2018, 13, 226-236. DOI: 10.1007/s12247-018-9317-1.

30. Guidelines for the Design and Evaluation of Oral Prolonged Release Dosage Forms; Ministry of Health, Labour, and Welfare: Tokyo, Japan, March 11, 1988. 